\title{
Predicting Remission Remains a Challenge in Patients with Juvenile Idiopathic Arthritis
}


The consequences of persistent active disease in juvenile idiopathic arthritis (JIA) include chronic pain and disability, in addition to growth disturbances and joint damage ${ }^{1,2}$. The last 2 decades have seen the development and licensing of biological therapies for JIA, revolutionizing patient care ${ }^{3}$. Now, more than ever, resolution of the signs and symptoms of JIA (i.e., remission) may be an attainable goal. However, even in cohorts of children and young people (CYP) with JIA where these newer therapies are widely available, fewer than $50 \%$ of CYP achieve remission in the first 10 years following diagnosis ${ }^{4}$.

For outcomes to improve in JIA, clinicians must take advantage of the window of opportunity. This window represents a short time after diagnosis whereby early treatments may be most effective ${ }^{5}$. Thus, appropriate therapies must be used as early as possible. There is an ongoing push toward stratified or personalized medicine across specialties, including rheumatology ${ }^{6}$. If nonremission could be predicted, patients at higher risk could be managed differently, for example, with the earlier use of targeted therapies such as biologics. This would additionally minimize the risk of adverse events from exposure to unnecessary therapies that may be less successful at controlling disease in that patient. However, it is currently unclear which patients are predisposed toward a remission-like course and which are at higher risk of maintaining active disease in the longer term.

Previous research has shown that the strongest predictor of remission in JIA is International League of Associations for Rheumatology (ILAR) category, with the oligoarticular ILAR category consistently associated with greater achievement and rheumatoid factor-positive polyarticular JIA with the lowest achievement of remission ${ }^{7}$. While this information is helpful for guiding general discussions with patients, it does not allow the calculation of an individual risk of nonremission given multiple patient characteristics. In this issue of The Journal, Guzman, et al present predictive models for nonremission in JIA using data from the Research in the Arthritis in Canadian Children emphasizing Outcomes study $(\text { ReACCh-Out })^{8}$. The authors aimed to produce statistical models that could accurately predict each patient's probability of remission within the first year following diagnosis. They considered clinical and demographic factors, and an a priori cutpoint was set for predictive ability of the model that would render it acceptable for use in clinical practice. Unfortunately, no models in the analysis were able to meet this cutpoint, showing that it is challenging to accurately predict which CYP will maintain active disease from diagnosis based on clinical factors alone.

Although the models did not meet the threshold for use in clinical practice, the study confirmed again that the strongest predictor of remission at diagnosis is ILAR category. With additional biomarkers such as antinuclear antibody, and patient-reported variables such as pain, about double the number of CYP could be classified as having high risk of nonremitting disease. This highlights the additional value of the patient/parent voice as well as laboratory biomarkers in predicting outcome.

The input of the patient/parent voice may have been an even stronger predictor of nonremission if this outcome had been defined according to the newer Juvenile Arthritis Disease Activity Score (JADAS) cutoffs ${ }^{9,10}$. The JADAS definition of remission includes a patient assessment of well-being and in this way differs from that used by Guzman, et $a l^{8}$, who used a modified version of the American College of Rheumatology criteria ${ }^{11}$. In the study, remission was defined as no active joints, normal erythrocyte sedimentation rate/C-reactive protein, a score $<1 \mathrm{~cm}$ on a $10-\mathrm{cm}$ physician's global assessment, morning stiffness not exceeding $15 \mathrm{~min}$, and no extraarticular features of systemic JIA, enthesitis-related JIA, or uveitis including no use of corticosteroid eye drops. This modified definition allowed for greater validity in enthesitis-related arthritis by including enthesitis activity in the definition and avoided the known endpoint aversion observed when using physician visual analog scales ${ }^{12}$ by allowing scores under $1 \mathrm{~cm}$ to indicate remission, as opposed to the original $0-\mathrm{cm}$ requirement. However, previous research has shown that different groups of CYP are identified as

See Predicting remission in JIA, page 628

Personal non-commercial use only. The Journal of Rheumatology Copyright @ 2019 . All rights reserved. 
being in remission by definitions that differ in the extent to which a patient assessment of disease is included ${ }^{13}$. Where such patient assessment contributes to being "in remission," CYP have better longterm outcomes than if remission was only fulfilled using physician assessments ${ }^{14}$. While the definition adapted by Guzman, et al therefore represents the presence of persistent inflammatory disease activity and thus, perhaps, a continued need for escalating disease-modifying antirheumatic drug therapies, the groups identified as at high risk of not achieving remission may not be distinct regarding longer-term physical function or well-being ${ }^{8}$.

This paper by Guzman, et $\mathrm{al}^{8}$ adds to a previous publication from the same group that looked at predicting a severe disease course $^{15}$. This previous outcome encompassed a more holistic picture of disease, including components measured by both physicians and patient/parents. Unlike the current model for nonremission, the risk of severe disease could be correctly classified in a high number of CYP ${ }^{15}$. Patient-reported outcomes may therefore be less challenging to predict from the outset of diagnosis than inflammatory processes.

Further information is needed to improve prediction models for remission in JIA. The Nordic JIA group recently published an alternative prediction model for remission, using similar predictors but from 6 months following disease onset rather than at point of diagnosis ${ }^{16}$. They assessed these predictors against an outcome of remission off medication, using a slightly different definition of remission after a followup of 8 years. The model produced had moderate to high predictive ability. The differences in predictive ability compared with those produced by Guzman, et $a l^{8}$ could suggest that there are different predictors of these different modified definitions of remission. It may also be that a CYP response to treatment over the first 6 months of disease may be the most important factor for predicting longer-term outcomes, thus again supporting the concept of a window of opportunity. It should be noted that the 2 cohorts differed in their timing of recruitment, with the ReACCh-Out study using a patient selection window of 2005 to 2010 compared with the Nordic JIA cohort, which included patients with disease onset between 1997 and 2000. External validation is therefore required for both existing models in additional clinical cohorts, with refinement needed for individual patient subgroups. Further, additional factors, potentially including other biomarkers or factors collected later in the disease are needed to improve predictive abilities. Currently, however, consistently and accurately predicting remission in JIA remains a challenge.

\section{STEPHANIE J.W. SHOOP-WORRALL $(\mathbb{B}$,}

$\mathrm{PhD}, \mathrm{MSc}, \mathrm{BSc}$,

Arthritis Research UK Centre for Epidemiology,

and Health eResearch Centre,

The University of Manchester;
KIMME L. HYRICH (D), MD, PhD, FRCPC,

Arthritis Research UK Centre for Epidemiology, The University of Manchester, and the UK National Institute for Health Research Manchester Biomedical Research Centre, Manchester University Hospitals

National Health Service Foundation Trust, Manchester Academic Health Science Centre, Manchester, UK.

Address correspondence to Prof. K.L. Hyrich, 2.800 Stopford Building, The University of Manchester, Oxford Road, Manchester M13 9PT, UK. E-mail: Kimme.hyrich@manchester.ac.uk

\section{REFERENCES}

1. Ravelli A, Martini A. Juvenile idiopathic arthritis. Lancet 2007;369:767-78.

2. Oliveira-Ramos F, Eusebio M, Martins M, Mourao AF, Furtado C, Campanilho-Marques R, et al. Juvenile idiopathic arthritis in adulthood: fulfilment of classification criteria for adult rheumatic diseases, long-term outcomes and predictors of inactive disease, functional status and damage. RMD Open 2016;2:e000304.

3. James RA, Wedderburn LR. Modern management of juvenile idiopathic arthritis. Prescriber 2016;27:37-43.

4. Shoop-Worrall SJW, Kearsley-Fleet L, Thomson W, Verstappen SMM, Hyrich KL. How common is remission in juvenile idiopathic arthritis: A systematic review. Semin Arthritis Rheum 2017; 47:331-7.

5. Stoll ML, Cron RQ. Treatment of juvenile idiopathic arthritis: a revolution in care. Pediatr Rheumatol Online J 2014;12:13.

6. Medical Research Council. The MRC Framework for the Development, Design and Analysis of Stratified Medicine Research. [Internet. Accessed November 13, 2018.] Available from: https://mrc.ukri.org/publications/browse/ mrc-framework-for-stratified-medicine

7. Glerup M, Herlin T, Twilt M. Clinical outcome and long-term remission in JIA. Curr Rheumatol Rep 2017;19:75.

8. Guzman J, Henrey A, Loughin T, Berard RA, Shiff NJ, Jurencak R. Predicting which children with juvenile idiopathic arthritis will not attain early remission with conventional treatment: results from the ReACCh-Out cohort. J Rheumatol 2019;46:628-35.

9. Consolaro A, Bracciolini G, Ruperto N, Pistorio A, Magni-Manzoni $\mathrm{S}$, Malattia C, et al; Paediatric Rheumatology International Trials Organization. Remission, minimal disease activity, and acceptable symptom state in juvenile idiopathic arthritis: defining criteria based on the juvenile arthritis disease activity score. Arthritis Rheum 2012;64:2366-74.

10. Consolaro A, Negro G, Gallo MC, Bracciolini G, Ferrari C, Schiappapietra B, et al. Defining criteria for disease activity states in non-systemic juvenile idiopathic arthritis based on a three-variable juvenile arthritis disease activity score. Arthritis Care Res 2014;66:1703-9.

11. Wallace CA, Giannini EH, Huang B, Itert L, Ruperto N. American College of Rheumatology provisional criteria for defining clinical inactive disease in select categories of juvenile idiopathic arthritis. Arthritis Care Res 2011;63:929-36.

12. Taylor J, Giannini EH, Lovell DJ, Huang B, Morgan EM. Lack of concordance in interrater scoring of the provider's global assessment of children with juvenile idiopathic arthritis with low disease activity. Arthritis Care Res 2018;70:162-6.

13. Shoop-Worrall SJW, Verstappen SMM, Baildam E, Chieng A, Davidson J, Foster $\mathrm{H}$, et al. How common is clinically inactive disease in a prospective cohort of patients with juvenile idiopathic arthritis? The importance of definition. Ann Rheum Dis 2017;76:1381-8. 
14. Shoop-Worrall SJW, Verstappen SMM, McDonagh JE, Baildam E, Chieng A, Davidson J, et al. Long-term outcomes following achievement of clinically inactive disease in juvenile idiopathic arthritis: the importance of definition. Arthritis Rheumatol 2018;70:1519-29.

15. Guzman J, Henrey A, Loughin T, Berard RA, Shiff NJ, Jurencak R, et al; ReACCh-Out Investigators. Predicting which children with juvenile idiopathic arthritis will have a severe disease course: Results from the ReACCh-Out cohort. J Rheumatol 2017;44:23-40.
16. Rypdal V, Arnstad ED, Aalto K, Berntson L, Ekelund M, Fasth A, et al. Predicting unfavorable long-term outcome in juvenile idiopathic arthritis: results from the Nordic Cohort Study. Arthritis Res Ther 2018;20:91.

J Rheumatol 2019;46:552-4; doi:10.3899/jrheum.181245 